\title{
The Circulation in Patients with Chronic Bronchitis and Emphysema at Rest and during Exercise, with Special Reference to the Influence of Changes in Blood Viscosity and Blood Volume on the Pulmonary Circulation *
}

\author{
N. Segel ANd J. M. Bishop $\dagger$ \\ (From the Department of Medicine, University of Birmingham, Queen Elizabeth Hospital, \\ Birmingham, England)
}

It is widely held that increased blood viscosity due to polycythemia is an important factor contributing to pulmonary hypertension in patients with chronic bronchitis (1-5). An increase in the viscosity factor in Poiseuille's equation will increase the pressure drop across a vascular bed proportionately, provided volume flow and the dimensions of the vessels remain constant. Roos (6) showed, in the isolated lung of a dog, that increasing packed cell volume is associated with an increase in the pressure drop across the pulmonary vascular bed, blood flow being maintained constant. However, the effects of alterations in blood viscosity upon pulmonary intravascular pressures in man have not been established.

Baldwin, Cournand, and Richards (7) noted that the association of polycythemia with chronic bronchitis is rare. It has been shown that red cell volume is often slightly increased in patients with chronic bronchitis who have arterial desaturation $(8,9)$, but judging by the hematocrit or hemoglobin values in other reports of large numbers of patients (10-12), substantial polycythemia is an uncommon complication. The present investigation is concerned with a highly selected group of patients with chronic bronchitis complicated by marked secondary polycythemia. They were drawn from a large population of patients with chronic bronchitis who were seen in an area where the disease is especially common.

* Submitted for publication April 4, 1966; accepted June 23, 1966.

$\dagger$ Address requests for reprints to Dr. J. M. Bishop, Queen Elizabeth Hospital, Edgbaston, Birmingham 15, England.
First we have assessed the severity of the changes in the pulmonary circulation and in the arterial blood gas tensions at rest and during exercise in such patients.

Secondly, we have examined the influence of increased blood viscosity and increased blood volume, and the interrelationship of these two factors, on the pulmonary and systemic circulations in patients with chronic bronchitis at rest and during exercise. To this end, we compared measurements made before and after repeated venesections in the patients with marked polycythemia.

Thirdly, when it became evident that chronic hypervolemia plays a part in the observed circulatory changes, we decided to investigate the effects of acute expansion and reduction of the blood volume in another group of patients with chronic bronchitis whose blood volume and hematocrit were initially normal.

\section{Methods}

Patients. A total of 21 patients with chronic bronchitis were studied, this diagnosis being based upon a history of long standing productive cough and moderate or severe dyspnea. Fifteen of the patients had marked polycythemia and pulmonary hypertension; their physical characteristics are given in Table I. Each of these patients was in congestive failure when first seen, but was studied only after an initial period of bed rest, when signs of edema and congestive failure had disappeared and when, despite being ambulant again, weight had remained constant for at least 1 week. All the patients were receiving digoxin and chlorothiazide at the time of the study. In 13 patients (excluding B9 and B10) the electrocardiogram showed changes typical of right ventricular hypertrophy (13). During the 5 years of study, 11 of these patients are known to have died. 
Five patients did not have polycythemia and had never been in congestive failure. These patients were studied after recovery from exacerbations of respiratory infections. One patient was studied during congestive failure and again after recovery.

Technical methods. Red cell volume was measured with ${ }^{51} \mathrm{Cr}$-labeled erythrocytes, by the technique of Sterling and Gray (14) as modified by Mollison and Veall (15), and was calculated from the mean radioactivity of blood samples taken at 10,15 , and 20 minutes. Blood viscosity was estimated by Pirofsky's method (16), which entails measuring the pressure and flow across a calibrated needle inserted into an antecubital vein with the arm dependent. Hematocrit was measured on arterial blood and corrected for trapped plasma.

An indwelling needle was placed in the brachial artery, and a double lumen catheter was advanced to the lung so that its tip was wedged into a peripheral pulmonary artery while the proximal orifice lay in the pulmonary arterial trunk. In all but 3 patients, a catheter was also placed in the right atrium. The intravascular pressures were measured by capacitance manometers and recorded by a multichannel direct writing instrument. The zero reference level for pressure was $10 \mathrm{~cm}$ above the plane of the table on which the patient was lying. Mean pressures were determined by planimetry, and all pressures were averaged over at least three respiratory cycles.

Cardiac output was measured by the direct Fick method. Expired gas was collected in a Tissot spirometer over a period of 3 minutes and analyzed by the Scholander method. During this time, two samples each of arterial and mixed venous blood were taken for the spectrophotometric determination of percentage oxygen saturation (17). Blood oxygen capacity was determined photometrically as oxyhemoglobin, the method being regularly calibrated against estimates with the Van Slyke manometric apparatus.

Central blood volume was measured with a continuous fractional sampling device (18). Forty $\mu \mathrm{c}$ of human serum albumen labeled with ${ }^{181} \mathrm{I}$ (HSA- $\left.{ }^{131} \mathrm{I}\right)$ was rapidly injected into the pulmonary artery, and brachial arterial samples were collected at 0.5 -second intervals for 30 seconds into a series of heparinized tubes fixed in a rotating disc. The radioactivity in each sample of blood was plotted against time on semilogarithmic paper, and from the resulting curve the central blood volume and mean transit time were calculated by the Stewart-Hamilton method (19). The time required for the blood to traverse the connecting tube from the arterial needle to the rotating disc and the time for injection were subtracted from the observed mean transit time. Plasma volume was measured from the $\mathrm{HSA}^{-181}{ }^{12}$ concentrations after the central blood volume estimation, $5-\mathrm{ml}$ samples being taken at 10,15 , and 20 minutes after initial injection. The radioactivity was plotted against time on semilogarithmic paper, and the line best fitting the points was extrapolated back to the time of injection.

Arterial oxygen tension $\left(\mathrm{PaO}_{2}\right)$ was measured polarographically with the Clark electrode (20) and the car- bon dioxide tension $\left(\mathrm{PaCO}_{2}\right)$ by the interpolation method of Astrup (21).

Pulmonary vascular resistance was calculated in the usual way (22). Pulmonary vascular hindrance, which characterizes the opposition to flow by the vascular system due to its dimensions as distinct from the opposition to flow derived from the fluid itself, was calculated as the pulmonary vascular resistance divided by the viscosity and expressed in arbitrary units (23).

Procedure. All measurements were made with the patient in the supine position, without sedation, and 6 hours after he had eaten a light meal. In the studies of the patients with polycythemia, the measurements were repeated 7 to 10 days later, during which time $500 \mathrm{ml}$ of blood was removed on alternate days until the hematocrit was reduced to the normal range. The final venesection was done not later than 36 hours before the second study.

On each occasion, the blood viscosity and hematocrit were first measured in duplicate. Central blood volume and plasma volume were then measured, and this was followed by estimation of red cell volume. These procedures occupied about 60 minutes, and then the cardiac output was measured at rest and again during the fourth minute of exercise on a bicycle ergometer. Intravascular pressures were recorded immediately before and after each estimate of cardiac output. The exercise performed during the second study was adjusted to be equal in severity to the first, and as judged by the oxygen uptake, this was usually achieved.

The procedure in the second series of patients without polycythemia will be apparent from Figure 5. After measurements of blood viscosity, packed cell volume, cardiac output, and intravascular pressures had been made, a rapid infusion of $25 \mathrm{~g} \mathrm{HSA}$, dissolved in $500 \mathrm{ml}$ $5 \%$ dextrose and warmed to body temperature, was given into the right atrium over 10 minutes. The same cycle of measurements was repeated immediately after the infusion had been completed, again after a rapid venesection $(500 \mathrm{ml})$ over 15 minutes, and finally after a second infusion, similar to the first. Observations in our laboratory have shown that the expansion of blood volume after an infusion of HSA remains fairly constant for at least 50 minutes.

Normal values. In selecting normal values with which to compare the results we have obtained, we have had to take note of the advanced age of some of our patients and to choose comparable normal data wherever possible.

For blood volume we have used the results from older male subjects of Yiengst and Shock (24) and of Hart and Metz (25), who used similar methods. The mean values are as follows: red cell volume, $33.3 \mathrm{ml}$ per $\mathrm{kg}$ (SD 0.7) ; plasma volume, $44.5 \mathrm{ml} \mathrm{per} \mathrm{kg}$ (SD 0.8); and total blood volume, $82.3 \mathrm{ml}$ per $\mathrm{kg}$ (SD 2.0). We have taken the mean hematocrit (uncorrected for trapped plasma) to be $45.2 \%$ (SD 2.63) according to Wennesland and co-workers (26). We have found a mean normal value for blood viscosity in 10 subjects of 2.23 centipoises (SD 0.31). Values for cardiac output in older subjects have been taken from the work of Brandfonbrener, Lan- 
downe, and Shock (27) and Granath, Jonsson, and Strandell (28). Since the latter authors and Dexter, Haynes, and Smith (29) have shown that advancing age has no effect upon the pulmonary intravascular pressures, we have used our own results for these parameters (30). The estimates of central blood volume were compared with those of Doyle, Wilson, Lépine, and Warren (31) and Lilienfield and associates (32).

Normal valués for cardiac output, intravascular pressures, and pulmonary ventilation during exercise were taken from the results of Donald, Bishop, Cumming, and Wade (33) and Granath and associates (28), the latter in older subjects.

Normal values for the partial pressure of oxygen and carbon dioxide in arterial blood have been predicted from the results of Raine and Bishop (34).

\section{Results}

At rest before venesection. The results are given in Table I. The hematocrit fell within the normal range in 1 patient only, but the red cell volume was greater than normal in all patients. Plasma volume was within the normal range in all patients, but total blood volume was normal in only 6 and abnormally high in the remaining 8 patients. The ratio of body to venous hematocrit was normal, ranging from 0.875 to 1.00 (mean 0.926 ). Blood viscosity was greater than normal in all patients.

Arterial oxygen tension was less than predicted in all but 1 of the patients, and the percentage oxygen saturation was similarly reduced. Arterial carbon dioxide tension was greater than normal in all but 1 patient (B9) in whom the oxygen tension was also normal.

The cardiac output fell within the normal range, but the mean heart rate was increased and the stroke volume reduced. There was no significant difference between cardiac output measured simultaneously in 11 of the patients by the Fick and HSA- ${ }^{131}$ I methods. Central blood volume was on the average greater than normal, but represented a normal proportion of total blood volume $(21.3 \%)$. There was a significant positive correlation between central blood volume and total blood volume $(r=0.681, p<0.05)$. The mean transit time was greater than normal (mean 13.9 seconds, SD 4.0). The mean oxygen uptake, pulmonary ventilation, and arteriovenous oxygen difference were all slightly greater than normal.

Pulmonary wedge pressure exceeded the upper limit of the normal range in 3 patients, and right atrial pressure was abnormally high in most of those in whom it was measured. Pulmonary arterial pressure was greater than normal in all patients, and most had severe pulmonary hypertension. Pulmonary vascular resistance and pulmonary vascular hindrance were greatly increased, but systemic vascular resistance was normal.

The relationships of pulmonary arterial mean pressure to certain other measurements have been examined, and the correlation coefficients are shown in Table II. There was a positive correlation with red cell volume and total blood volume and with $\mathrm{Pa}_{\mathrm{CO}_{2}}$, and a negative correlation with arterial oxygen saturation and $\mathrm{Pa}_{\mathrm{O}_{2}}$. A multiple regression analysis gave the following equation: $\mathrm{PAP}=65.333+0.126(\mathrm{TBV})-0.582\left(\mathrm{~Pa}_{\mathrm{O}_{2}}\right)$, where PAP is pulmonary arterial mean pressure (millimeters $\mathrm{Hg}$ ), $\mathrm{TBV}$ is total blood volume (milliliters per kilogram), and $\mathrm{Pa}_{\mathrm{O}_{2}}$ is in millimeters $\mathrm{Hg}$. There was not significant improvement in the prediction when $\mathrm{Pa}_{\mathrm{CO}_{2}}$ was included, either before or after venesection.

At rest after repeated venesection. The results after repeated venesection, given in Table I, show a significant reduction in hematocrit, red cell volume, and blood viscosity, and these values now fell within the normal range in all but 1 patient (B6). The plasma volume expanded by varying degrees, and the average value after venesection was significantly greater than before. In 6 patients (B1 to B6), the total blood volume decreased by more than $6.0 \mathrm{ml}$ per $\mathrm{kg}$, whereas in the others, the compensatory increase in plasma volume was sufficient to maintain an essentially unchanged total blood volume. $\mathrm{Pa}_{2}$ and $\mathrm{Pa}_{\mathrm{CO}_{2}}$ showed no consistent changes after venesection.

There was no significant change in cardiac output, heart rate, or stroke volume after venesection. There was a small but significant fall in central blood volume, the reduction being greatest in those patients in whom total blood volume also decreased. There was a positive correlation between the change in central and total blood volumes $(r=0.883, p<0.001)$, and central blood volume as a proportion of total blood volume $(21.8 \%)$ remained unchanged. Mean transit time decreased significantly (mean 11.3 seconds, SD 2.5). The average oxygen uptake, pulmonary ventilation, and arteriovenous oxygen difference were unaffected by venesection. 
TABLE I

Individual values for cardiac output and related measurements, intravascular pressures,

\begin{tabular}{|c|c|c|c|c|c|c|c|c|c|c|c|c|}
\hline$\underset{\text { Age }}{\text { Subject }}$ & Sex & $\mathbf{W t}$ & BSA & Period & $\begin{array}{l}\text { Oxygen } \\
\text { uptake }\end{array}$ & $\begin{array}{c}\text { Pulmo- } \\
\text { nary ven- } \\
\text { tilation }\end{array}$ & $\begin{array}{l}\text { Cardiac } \\
\text { output }\end{array}$ & $\begin{array}{l}\text { Heart } \\
\text { rate }\end{array}$ & $\begin{array}{c}\text { Central } \\
\text { blood } \\
\text { volume }\end{array}$ & $\begin{array}{c}\text { Arterial } \\
\text { oxygen } \\
\text { saturation }\end{array}$ & $\mathrm{PaO}_{2}{ }^{*}$ & $\mathrm{PaCO}_{2} \dagger$ \\
\hline years & & $\mathrm{kg}$ & $m^{2}$ & & $\begin{array}{c}m l / m i n / \\
m^{2}\end{array}$ & $\underset{m^{2}}{L / \min /}$ & $\underset{m^{2}}{L / \min /}$ & & $L / m^{2}$ & $\%$ & $m m H g$ & $m m \mathrm{Hg}$ \\
\hline B1 & $F$ & 77.7 & 1.78 & Before & 156 & 5.0 & 3.8 & 96 & 0.64 & 78.3 & 50.0 & 60.1 \\
\hline 60 & & 77.5 & 1.79 & After & 148 & 4.2 & 3.3 & 83 & 0.53 & 88.6 & 60.1 & 46.5 \\
\hline B2 & $\mathbf{M}$ & 61 & 1.65 & Before & 149 & 5.9 & 2.7 & 89 & 0.59 & 92.6 & 67.7 & 48.2 \\
\hline 64 & & 62 & 1.66 & After & 145 & 5.5 & 2.9 & 92 & 0.53 & 90.5 & 66.9 & 49.9 \\
\hline B3 & $\mathbf{M}$ & 53 & 1.54 & Before & 158 & 4.7 & 2.8 & 99 & 0.72 & 69.6 & 36.5 & 69.2 \\
\hline 48 & & 52 & 1.52 & After & 149 & 4.8 & 3.0 & 100 & 0.57 & 74.8 & 44.7 & 58.4 \\
\hline B4 & $\mathbf{M}$ & 53 & 1.63 & Before & 110 & 4.7 & 3.4 & 104 & 0.84 & 73.5 & 42.9 & 70.6 \\
\hline 51 & & 51 & 1.60 & After & 108 & 5.4 & 3.7 & 108 & 0.61 & 79.5 & 50.9 & 72.9 \\
\hline B5 & $\mathbf{M}$ & 83 & 2.01 & Before & 163 & 4.3 & 4.2 & 115 & & 53.7 & 29.4 & 64.7 \\
\hline 61 & & 84 & 2.01 & After & 157 & 4.0 & 4.0 & 119 & & 65.0 & 43.8 & 71.4 \\
\hline B6 & $\mathbf{M}$ & 50 & 1.57 & Before & 189 & 4.1 & 4.3 & 110 & & 71.3 & 35.0 & 81.0 \\
\hline 29 & & 51.5 & 1.60 & After & 181 & 4.2 & 3.5 & 120 & & 63.4 & 29.9 & 91.9 \\
\hline B7 & $\mathbf{M}$ & 57 & 1.71 & Before & 164 & 4.4 & 3.5 & 93 & 0.70 & 74.4 & 53.1 & 55.0 \\
\hline 30 & & 55.5 & 1.70 & After & 157 & 4.2 & 4.6 & 95 & 0.74 & 75.9 & 53.0 & 57.9 \\
\hline B8 & $\mathbf{M}$ & 83.5 & 2.06 & Before & 169 & 4.1 & 3.4 & 92 & 0.60 & 86.8 & 59.8 & 57.5 \\
\hline 58 & & 86.5 & 2.10 & After & 162 & 4.2 & 4.6 & 93 & 0.58 & 84.3 & 52.8 & 60.1 \\
\hline B9 & $\mathbf{M}$ & 58 & 1.65 & Before & 159 & 7.8 & 2.6 & 91 & 0.74 & 89.0 & 78.2 & 32.6 \\
\hline 64 & & 58 & 1.65 & After & 126 & 6.4 & 2.3 & 89 & 0.70 & 89.5 & 78.5 & 33.4 \\
\hline B10 & $\mathbf{M}$ & 74.5 & 1.76 & Before & 148 & 4.1 & 2.9 & 77 & 0.67 & 89.5 & 65.4 & 48.5 \\
\hline 41 & & 72.5 & 1.74 & After & 150 & 3.8 & 2.6 & 70 & 0.63 & 92.3 & 72.4 & 47.9 \\
\hline B11 & $\mathbf{M}$ & 57 & 1.70 & Before & 155 & 6.0 & 3.3 & 105 & 0.70 & 90.8 & 53.0 & 48.5 \\
\hline 50 & & 56.5 & 1.70 & After & 136 & 6.2 & 3.2 & 88 & 0.65 & 88.0 & 54.0 & 45.5 \\
\hline B12 & $\mathbf{M}$ & 51.5 & 1.60 & Before & 126 & 3.8 & 2.9 & 90 & 0.72 & 86.3 & 53.7 & 56.8 \\
\hline 56 & & 51 & 1.58 & After & 156 & 4.7 & 2.9 & 89 & 0.78 & 82.0 & 43.1 & 59.0 \\
\hline B13 & $\mathbf{M}$ & 57.5 & 1.64 & Before & 155 & 5.7 & 2.3 & 99 & 0.79 & 89.9 & 50.4 & 52.2 \\
\hline 53 & & 56.0 & 1.62 & After & 155 & 5.6 & 2.8 & 97 & 0.76 & 93.0 & 54.2 & 53.3 \\
\hline B14 & $\mathbf{M}$ & 67 & 1.69 & Before & 174 & 6.4 & 3.9 & 115 & & 85.9 & 51.9 & 56.0 \\
\hline 58 & & 67 & 1.69 & After & 146 & 6.6 & 5.3 & 106 & & 88.0 & 53.2 & 49.0 \\
\hline $\begin{array}{r}\text { B15 } \\
54\end{array}$ & $\mathbf{M}$ & 61 & 1.75 & Before & 144 & 5.5 & 3.7 & 92 & 0.69 & 89.1 & 61.9 & 50.0 \\
\hline \multirow{2}{*}{\multicolumn{2}{|c|}{$\begin{array}{l}\text { Mean } \\
\text { (B1-B14) }\end{array}$}} & & & Before & $\begin{array}{l}155 \\
(19.31) \ddagger\end{array}$ & $\begin{array}{c}4.1 \\
(1.15)\end{array}$ & $\begin{array}{c}3.3 \\
(0.58)\end{array}$ & $\begin{array}{l}98 \\
(9.86)\end{array}$ & $\begin{array}{c}0.70 \\
(0.08)\end{array}$ & $\begin{array}{c}81.2 \\
(11.45)\end{array}$ & $\begin{array}{c}51.9 \\
(13.32)\end{array}$ & $\begin{array}{c}57.2 \\
(11.85)\end{array}$ \\
\hline & & & & After & $\begin{array}{l}148 \\
(17.10)\end{array}$ & $\begin{array}{c}5.0 \\
(0.96)\end{array}$ & $\begin{array}{c}3.5 \\
(0.83)\end{array}$ & $\begin{array}{l}96 \\
(13.57)\end{array}$ & $\begin{array}{c}0.64 \\
(0.09)\end{array}$ & $\begin{array}{c}83.0 \\
(11.57)\end{array}$ & $\begin{array}{c}53.1 \\
(12.49)\end{array}$ & $\begin{array}{c}56.9 \\
(14.43)\end{array}$ \\
\hline p & & & & & $<0.10$ & $<0.10$ & $<0.10$ & $<0.10$ & $<0.05$ & $<0.10$ & $<0.10$ & $<0.10$ \\
\hline
\end{tabular}

* Arterial oxygen tension.

† Arterial carbon dioxide tension.

$\ddagger$ Standard deviation.

The average pulmonary wedge pressure showed no change, but in 4 of 6 patients in whom total blood volume fell, there was a decrease in wedge pressure. Right atrial pressure fell in all but one of the 11 patients in whom it was measured. There was a small but significant reduction in pulmonary arterial mean pressure in the group of patients. There was a positive correlation between the fall in pulmonary arterial pressure and the fall in blood volume $(\mathrm{r}=0.804, \mathrm{p}<0.001)$ (Figure 1 ), and in the patients in whom the latter did not fall, the pulmonary arterial pressure remained unaltered. A similar correlation was found with changes in central blood volume $(r=0.758, p<$ $0.01)$. Average pulmonary vascular resistance decreased, but pulmonary vascular hindrance increased to a value that was even more abnormal. The individual changes were greatest in patients 
TABLE I

arterial gas tensions, and blood volume at rest before and after repeated venesection

\begin{tabular}{|c|c|c|c|c|c|c|c|c|c|c|c|c|}
\hline \multicolumn{4}{|c|}{ Mean pressure } & \multirow{2}{*}{\multicolumn{2}{|c|}{ Vascular resistance }} & \multirow{2}{*}{\multicolumn{2}{|c|}{ Vascular hindrance }} & \multirow{3}{*}{$\begin{array}{l}\text { Packed } \\
\text { cell } \\
\text { volume }\end{array}$} & \multirow{2}{*}{\multicolumn{3}{|c|}{ Blood volume }} & \multirow[b]{3}{*}{ Viscosity } \\
\hline \multirow{2}{*}{$\begin{array}{c}\text { Bra- } \\
\text { chial } \\
\text { artery }\end{array}$} & \multirow{2}{*}{$\begin{array}{c}\text { Pul- } \\
\text { mo- } \\
\text { nary } \\
\text { artery }\end{array}$} & \multirow{2}{*}{$\begin{array}{c}\text { Pul- } \\
\text { mo- } \\
\text { nary } \\
\text { wedge }\end{array}$} & \multirow[b]{2}{*}{$\begin{array}{c}\text { Right } \\
\text { atrium }\end{array}$} & & & & & & & & & \\
\hline & & & & $\begin{array}{l}\text { Pul- } \\
\text { mo- } \\
\text { nary }\end{array}$ & $\begin{array}{l}\text { Sys- } \\
\text { temic }\end{array}$ & $\begin{array}{l}\text { Pul- } \\
\text { mo- } \\
\text { nary }\end{array}$ & $\begin{array}{c}\text { Sys- } \\
\text { temic }\end{array}$ & & $\begin{array}{l}\text { Red } \\
\text { cell }\end{array}$ & Plasma & Total & \\
\hline & \multicolumn{2}{|c|}{$m m \mathrm{Hg}$} & & \multicolumn{2}{|c|}{ dyne-sec-cm-6 } & & & $\%$ & & $m l / k g$ & & centipoises \\
\hline 115 & 50 & 19 & 15 & 364 & 1,351 & 61 & 225 & 64 & 46.3 & 30.5 & 76.8 & 6.0 \\
\hline 77 & 36 & 14 & 9 & 298 & 1,043 & 93 & $\mathbf{4 4 7}$ & 47 & 27.3 & 31.7 & 59.0 & 3.2 \\
\hline 123 & 46 & 12 & 5 & 604 & 2,184 & 96 & 347 & 60 & 44.0 & 38.3 & 82.3 & 6.3 \\
\hline 122 & 40 & 8 & 5 & 533 & 2,032 & 197 & 753 & 43 & 31.8 & 44.5 & 76.3 & 2.7 \\
\hline 108 & 60 & 10 & & 910 & 1,962 & 159 & 344 & 64 & 63.0 & 46.6 & 110.0 & 5.7 \\
\hline 101 & 50 & 9 & & 783 & 1,755 & 340 & 763 & 45 & 39.5 & 49.6 & 89.2 & 2.3 \\
\hline 116 & 52 & 12 & 8 & 581 & 1,686 & 94 & 272 & 64 & 69.0 & 41.5 & 110.5 & 6.2 \\
\hline 119 & 39 & 9 & 5 & 400 & 1,585 & 153 & 610 & 45 & 34.2 & 51.0 & 85.2 & 2.6 \\
\hline 146 & 60 & 18 & 18 & 400 & 1,389 & 68 & 232 & 64 & 52.0 & 36.5 & 88.5 & 6.0 \\
\hline 118 & 55 & 18 & 15 & 352 & 1,123 & 110 & 351 & 47 & 34.0 & 44.5 & 80.0 & 3.2 \\
\hline 94 & 58 & 8 & 9 & 600 & 1,121 & 85 & 158 & 72 & 68.2 & 40.5 & 108.7 & 7.1 \\
\hline 94 & 49 & 11 & 7 & 532 & 1,473 & 140 & 388 & 54 & 50.7 & 48.6 & 99.4 & 3.8 \\
\hline 105 & 50 & 8 & 6 & 547 & 1,399 & 96 & 245 & 55 & 51.2 & 46.3 & 97.5 & 5.7 \\
\hline 83 & 48 & 7 & 5 & 415 & 840 & 153 & 311 & 44 & 40.8 & 55.4 & 96.2 & 2.7 \\
\hline 108 & 48 & 9 & 12 & 439 & 1,216 & 98 & 270 & 51 & 39.5 & 42.6 & 82.1 & 4.5 \\
\hline 96 & 46 & 14 & 8 & 266 & 799 & 140 & 421 & 38 & 32.1 & 54.3 & 86.4 & 1.9 \\
\hline 121 & 28 & 4 & 9 & 446 & 2,249 & 86 & 433 & 54 & 44.0 & 37.4 & 81.5 & 5.2 \\
\hline 115 & 29 & 6 & 8 & 418 & 2,089 & 190 & 950 & 41 & 31.5 & 47.5 & 79.0 & 2.2 \\
\hline 107 & 29 & 13 & 12 & 246 & 1,645 & 48 & 323 & 58 & 39.6 & 37.3 & 75.0 & 5.1 \\
\hline 108 & 31 & 13 & 6 & 313 & 1,877 & 143 & 853 & 44 & 28.2 & 42.9 & 71.1 & 2.2 \\
\hline 110 & 42 & 11 & & 600 & 1,598 & 120 & 320 & 60 & 54.1 & 39.7 & 93.8 & 5.0 \\
\hline 118 & 40 & 15 & & 363 & 1,714 & 191 & 902 & 42 & 34.8 & 54.1 & 88.9 & 1.9 \\
\hline 123 & 36 & 8 & & 486 & 2,137 & 84 & 368 & 58 & 47.2 & 44.6 & 91.8 & 5.8 \\
\hline 104 & 42 & 9 & & 599 & 1,889 & 261 & 821 & 41 & 32.8 & 56.5 & 89.3 & 2.3 \\
\hline 114 & 55 & 17 & 7 & 800 & 2,398 & 148 & 444 & 60 & 51.5 & 43.5 & 95.0 & 5.4 \\
\hline 110 & 55 & 14 & 6 & 713 & 1,911 & 254 & 683 & 45 & 40.0 & 53.6 & 93.6 & 2.8 \\
\hline 120 & 38 & 11 & 9 & 322 & 1,431 & 57 & 251 & 62 & 47.0 & 37.4 & 84.4 & 5.7 \\
\hline 116 & 35 & 12 & 8 & 207 & 1,041 & 71 & 359 & 42 & 29.3 & 50.0 & 79.3 & 2.9 \\
\hline 102 & 41 & 12 & 6 & 376 & 1,255 & 64 & 213 & 63 & 47.4 & 42.4 & 89.8 & 5.9 \\
\hline $\begin{array}{l}115 \\
(12.02)\end{array}$ & $\begin{array}{l}47 \\
(10.66)\end{array}$ & $\begin{array}{l}11 \\
(4.23)\end{array}$ & $\begin{array}{l}10 \\
(3.92)\end{array}$ & $\begin{array}{l}514 \\
(179.5)\end{array}$ & $\begin{array}{l}1,698 \\
(415.7)\end{array}$ & $\begin{array}{l}93 \\
(32.0)\end{array}$ & $\begin{array}{l}302 \\
(81.1)\end{array}$ & $\begin{array}{l}60 \\
(5.26)\end{array}$ & $\begin{array}{l}51.2 \\
(9.56)\end{array}$ & $\begin{array}{l}40.2 \\
(4.40)\end{array}$ & $\begin{array}{l}91.3 \\
(12.00)\end{array}$ & $\begin{array}{l}5.7 \\
(0.63)\end{array}$ \\
\hline $\begin{array}{l}106 \\
(14.02)\end{array}$ & $\begin{array}{l}43 \\
(8.28)\end{array}$ & $\begin{array}{l}11 \\
(4.27)\end{array}$ & $\begin{array}{l}7 \\
(2.88)\end{array}$ & $\begin{array}{l}442 \\
(169.1)\end{array}$ & $\begin{array}{l}1,512 \\
(455.6)\end{array}$ & $\begin{array}{l}174 \\
(72.4)\end{array}$ & $\begin{array}{l}615 \\
(229.4)\end{array}$ & $\begin{array}{l}44 \\
(3.76)\end{array}$ & $\begin{array}{l}34.8 \\
(6.20)\end{array}$ & $\begin{array}{l}48.7 \\
(6.44)\end{array}$ & $\begin{array}{l}83.6 \\
(10.60)\end{array}$ & $\begin{array}{c}2.6 \\
(0.52)\end{array}$ \\
\hline$<0.02$ & $<0.02$ & & $<0.001$ & $<0.01$ & $<0.02$ & $<0.001$ & $<0.001$ & $<0.001$ & $<0.001$ & $<0.001$ & $<0.01$ & $<0.001$ \\
\hline
\end{tabular}

whose total blood volume decreased. There was a small but significant fall in mean systemic vascular resistance, whereas mean systemic vascular hindrance increased from 302 to $615 \mathrm{U}$, the latter being approximately normal.

The correlation coefficients between pulmonary arterial pressure and other parameters as determined after venesection are given in Table II. Multiple regression analysis gave the following relationship: $\mathrm{PAP}=34.144+0.297(\mathrm{TBV})-$ $0.311\left(\mathrm{~Pa}_{\mathrm{O}_{2}}\right)$.

During exercise before venesection. Individual results are given in Table III, and the relationship between cardiac output and oxygen uptake during exercise is illustrated in Figure 2. The cardiac output achieved during exercise was normal in 7 patients, but was less than normal in 8, being grossly impaired in 3 of these. As with 
TABLE II

Correlation coefficients among pulmonary arterial pressure and vascular resistance, blood volume, arterial oxygen saturation, and arterial gas tensions at rest and during exercise before and after repeated venesection*

\begin{tabular}{|c|c|c|c|c|c|}
\hline & $\mathrm{SaO}_{2}$ & $\mathrm{PaO}_{2}$ & $\mathrm{PaCO}_{2}$ & $\mathrm{RCV}$ & TBV \\
\hline \multicolumn{6}{|c|}{ At rest, before venesection } \\
\hline $\begin{array}{l}\text { PAP, } m m H g \\
\text { RCV, } m l / k g\end{array}$ & $\begin{array}{l}-0.676 \dagger \\
-0.5438\end{array}$ & $\begin{array}{l}-0.820 \dagger \\
-0.722 \dagger\end{array}$ & $\begin{array}{l}0.753 \dagger \\
0.745 t\end{array}$ & $0.631 \ddagger$ & $0.615 \ddagger$ \\
\hline PVR, dyne-sec-cm-5 & -0.105 & -0.329 & $\begin{array}{l}0.273 \mathrm{~T} \\
0.273\end{array}$ & 0.427 & $0.714 \dagger$ \\
\hline \multicolumn{6}{|c|}{ At rest, after venesection } \\
\hline $\mathrm{PAP}, \mathrm{mm} \mathrm{Hg}$ & $-0.680 \dagger$ & $-0.652 \ddagger$ & $0.578 \S$ & $0.658 \ddagger$ & $0.611 \S$ \\
\hline $\mathrm{RCV}, \mathrm{ml} / \mathrm{kg}$ & $-0.629 \ddagger$ & -0.5968 & $0.685 \dagger$ & & \\
\hline PVR, dyne-sec- $\mathrm{cm}^{-5}$ & -0.043 & -0.185 & 0.183 & $0.875 \dagger$ & 0.503 \\
\hline \multicolumn{6}{|c|}{ Exercise, before venesection } \\
\hline $\mathrm{PAP}, m m \mathrm{Hg}$ & $-0.518 \S$ & $-0.792 \dagger$ & $0.913 \dagger$ & & \\
\hline \multicolumn{6}{|c|}{ Exercise, after venesection } \\
\hline PAP, $m m ~ H g$ & $-0.663 \dagger$ & $-0.595 \S$ & $0.733 \ddagger$ & & \\
\hline
\end{tabular}

$* \mathrm{SaO}_{2}=$ arterial oxygen saturation; $\mathrm{PaO}_{2}=$ arterial oxygen tension; $\mathrm{PaCO}_{2}=$ arterial carbon dioxide tension; $\mathrm{RCV}=$ red cell volume; $\mathrm{TBV}=$ total blood volume $; \mathrm{PAP}=$ pulmonary arterial mean pressure; PVR = pulmonary vascular resistance.

tp 0.01 .

$\ddagger \mathrm{p} 0.02$.

$\$ \mathrm{p} 0.05$.

normal subjects exercising in the supine posture, the increase in cardiac output was the result of an increased heart rate, stroke volume remaining unchanged.

Arterial oxygen saturation decreased in all patients, usually to a marked degree, whereas carbon dioxide tension usually increased. Pulmonary ventilation increased to a normal extent in most patients, but was slightly greater than normal in 2 .

There was a large increase in pulmonary arterial mean pressure, and the average during exercise was $77 \mathrm{~mm} \mathrm{Hg}$ (range 66 to 86). Pulmonary wedge pressure also increased in most patients, the rise being greater than normal in several. Relationships between pulmonary arterial pres-

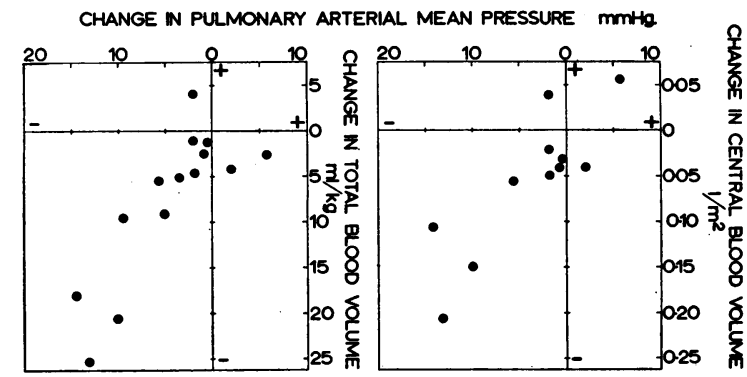

Fig. 1. INDIVIdUal VALUES FOR ChANGE IN PULMONARY ARTERIAL MEAN PRESSURE AND CHANGE IN TOTAL BLOOD VOLUME AND CENTRAL BLOOD VOLUME, AT REST, AFTER REPEATED VENESECTION. sure and arterial blood gas tensions during exercise were similar to those found at rest (Table II).

During exercise after venesection. The results are given in Table III. Cardiac output and its relationship to oxygen uptake are illustrated in Figure 2. The work done as judged by the oxygen uptake was on the average the same as before. The level of cardiac output achieved during exercise was more nearly normal in several patients than before venesection. Cardiac output during exercise was greater than before in 10 patients and less than before in only 2 . The heart rate increased to a similar extent with exercise, but the increase in stroke volume was a little greater, the change from a mean of $36 \mathrm{ml}$ per $\mathrm{m}^{2}$ at rest to 41 $\mathrm{ml}$ per $\mathrm{m}^{2}$ during exercise being significant $(\mathrm{p}<$ $0.05)$.

The fall in arterial oxygen saturation and the rise in carbon dioxide tension were similar to those before venesection, and pulmonary ventilation was not changed.

The average level of pulmonary arterial mean pressure during exercise was significantly less than before venesection although the increase with exercise was similar. The response of pulmonary wedge pressure was unaltered. The relationships between pulmonary arterial pressure and arterial blood gas tensions were similar to those before venesection (Table II). Pulmonary vascular resistance rose to a significantly lower level than before venesection.

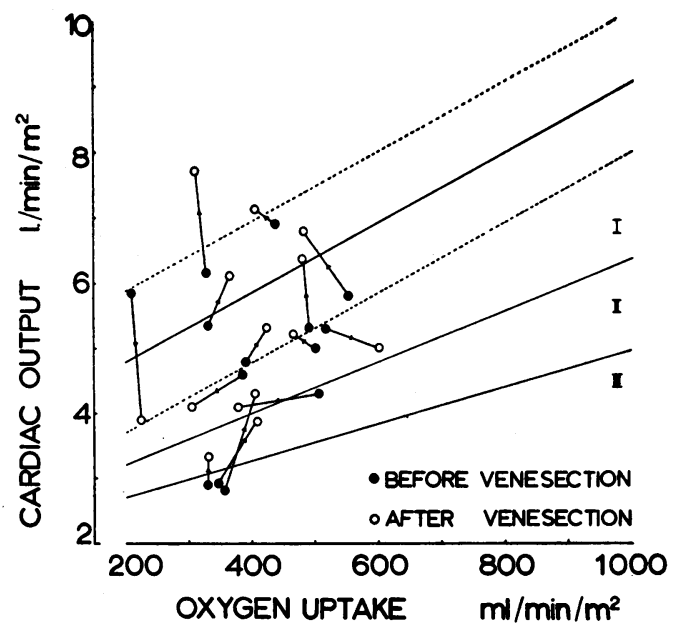

Fig. 2. INDIVIDUAL VALUES FOR CARDIAC OUTPUT AND OXYGEN UPTAKE DURING EXERCISE BEFORE AND AFTER VENESECTION. 


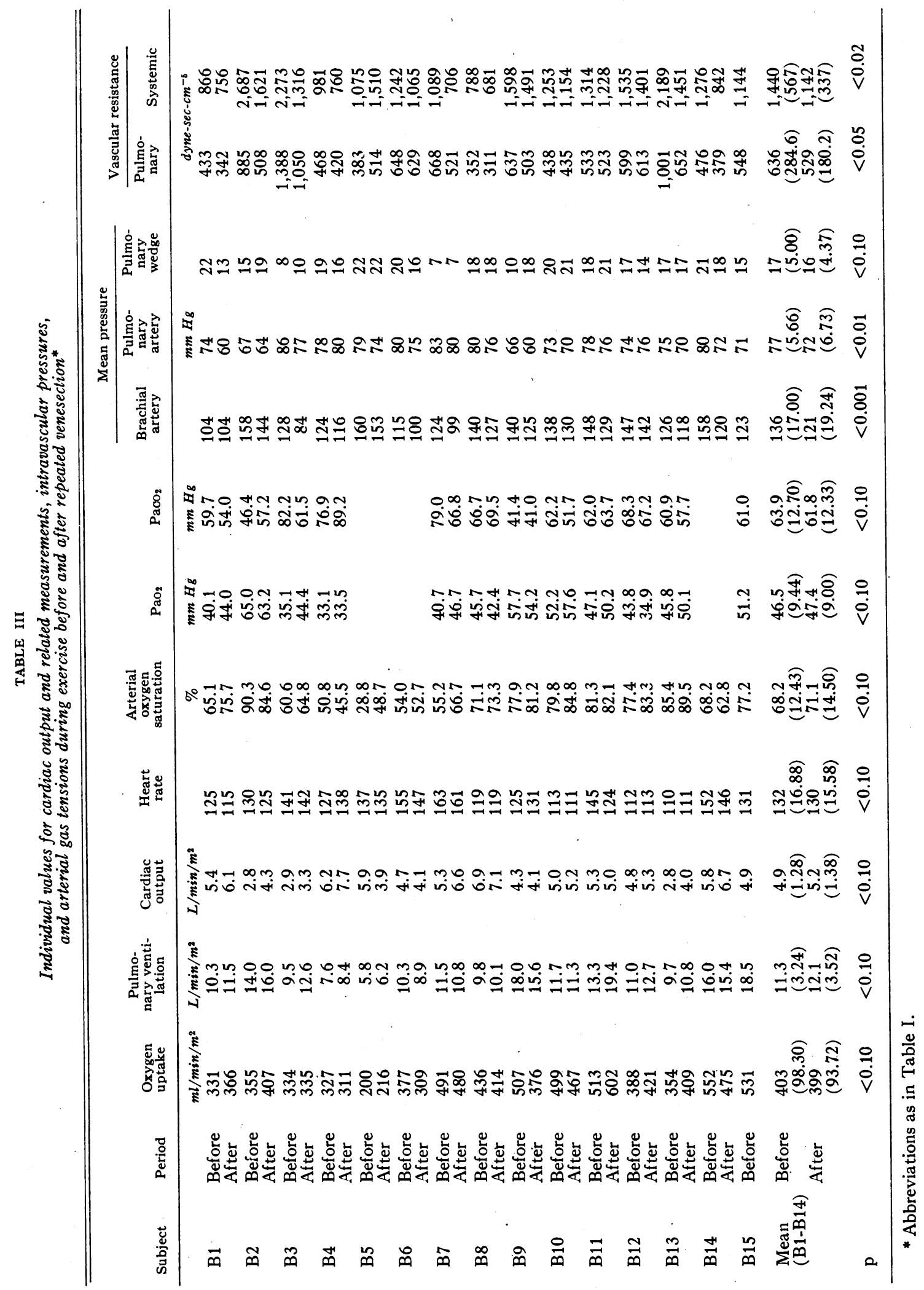




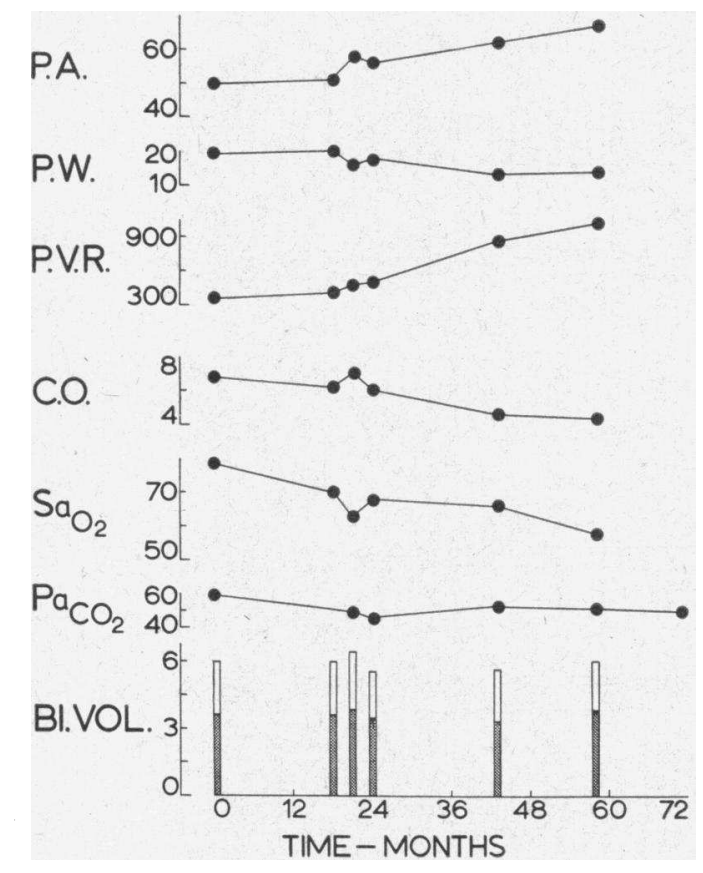

Fig. 3. Serial observations in Patient B1 of the MEAN PRESSURE (MILLIMETERS HG) IN THE PULMONARY ARTERY (PA) AND PULMONARY WEDGE (PW), PULMONARY VASCULAR RESISTANCE (PVR, DYNES PER SECOND PER CENTIMETER $^{-5}$ ), CARDIAC OUTPUT (CO, LITERS PER MINUTE), ARTERIAL OXYGEN SATURATION ( $\mathrm{Sa}_{2}$, PER CENT), CARBON DIOXIDE TENSION ( $\mathrm{PaCO}_{2}$, MILlIMETERS $\mathrm{Hg}$ ), AND BLOOD VOLUME (BL.VOL. LITERS; HATCHED AREA IS RED CELL VOLume, Clear area is plasma volume). The patient died 72 months after the first observation.

Serial observations in patients $B 1$ and B12. $\mathrm{Pa}-$ tient $\mathrm{B} 1$, the only female in the group with polycythemia, was studied on several occasions over a period of 5 years; the results at rest are shown in Figure 3. On each occasion the study was performed when the patient had fully recovered from an acute respiratory infection that was often associated with signs of congestive failure. She received digoxin and diuretics throughout the period of observation.

During the first 2 years, she had more than 40 venesections and experienced considerable relief from headache, tinnitus, and fatigue. The effect of venesection was short lived, and blood volume was always increased. The progressive rise in pulmonary arterial pressure and in pulmonary vascular resistance was associated with a progressive fall in cardiac output. Arterial oxygen saturation decreased progressively during the years of study, but gross hypercapnia was not present. There was a significant inverse correlation between pulmonary arterial pressure and arterial oxygen saturation when the results of all studies were pooled. Autopsy showed diffuse panacinar emphysema with narrowing of pulmonary arterioles less than $100 \mu$ in diameter. These arterioles had prominent circular muscle fibers, but the larger muscular pulmonary arteries were normal. The right ventricle was grossly hypertrophied and weighed 210 $\mathrm{g}$ (normal not more than $65 \mathrm{~g}$ ). Similar observations were made in Patient B12, and the results are shown in Figure 4.

Effects of acutely induced changes in blood volume. In 5 patients, blood volume was rapidly expanded by the infusion of $500 \mathrm{ml}$ of a solution of $\mathrm{HSA}$, and this was followed by a venesection of $500 \mathrm{ml}$ and a further infusion. The mean results are shown in Figure 5. Before the infusion, 3 of the patients had pulmonary hypertension, and 2 showed mild arterial unsaturation. Cardiac out-

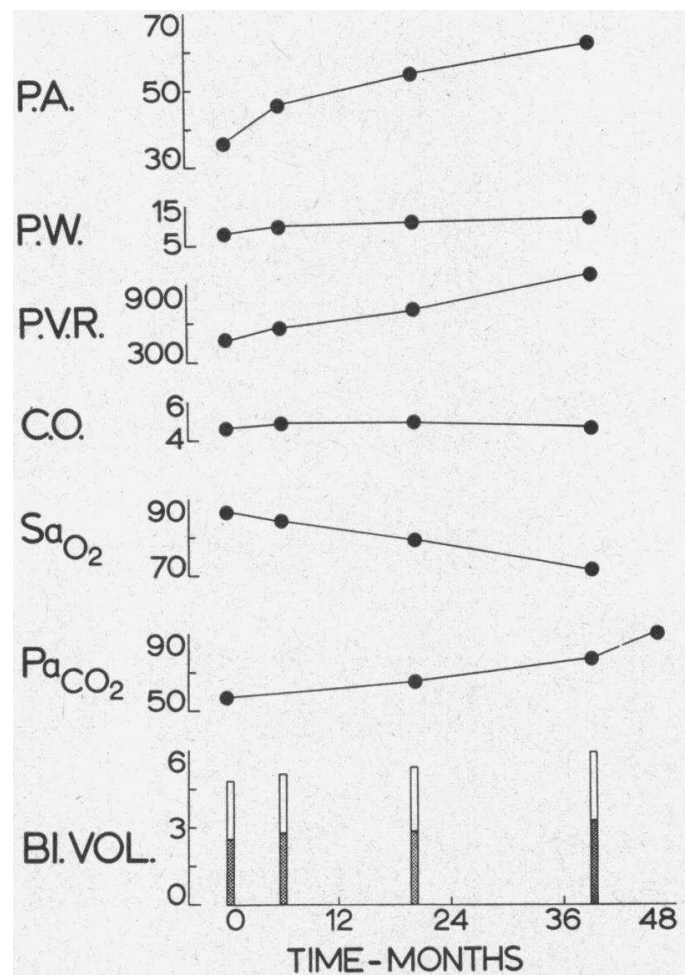

Fig. 4. Serial observations in Patient B12 of the MEAN PRESSURE IN THE PULMONARY ARTERY AND PULMONARY WEDGE, PULMONARY VASCULAR RESISTANCE, CARDIAC OUTPUT, ARTERIAL OXYGEN SATURATION, CARBON DIOXIDE TENSION, AND BLOOD VOLUME. The patient died 48 months after the first observation. See legend to Figure 3 for abbreviations. 
put, hematocrit, and blood viscosity were normal in all.

After the first infusion these was an increase in cardiac output and pulmonary arterial, pulmonary wedge, and right atrial pressures, whereas pulmonary vascular resistance remained unaltered. These changes were all reversed after venesection, when the values observed did not differ from the control values, the net gain in total blood volume having been corrected. The changes after the second infusion were similar to those after the first. Blood viscosity decreased progressively throughout the study, and the final values were significantly less than the initial one. Arterial oxygen saturation was unchanged throughout.

Effects of spontaneous reduction of blood volume. The findings are presented in Figure 6 of measurements made in 1 patient when congestive failure was present; these measurements were repeated 12 days later, when signs of congestive

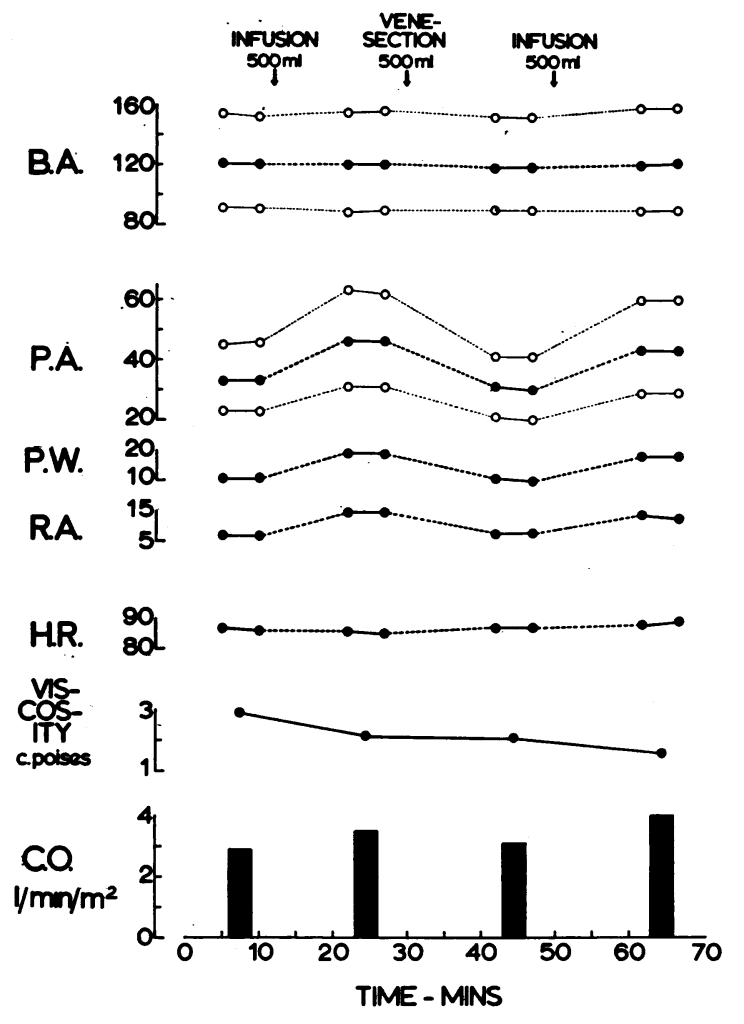

Fig. 5. EFFECT OF AN INFUSION OF HUMAN SERUM ALBUMEN AND VENESECTION ON THE PRESSURES (MILLIMETERS HG) IN THE BRACHIAL ARTERY (BA), PULMONARY ARTERY (PA), PULMONARY WEDGE (PW), AND RIGHT ATRIUM (RA), HEART RATE (HR), VISCOSITY, AND CARDIAC OUTPUT. Mean values for 5 patients.
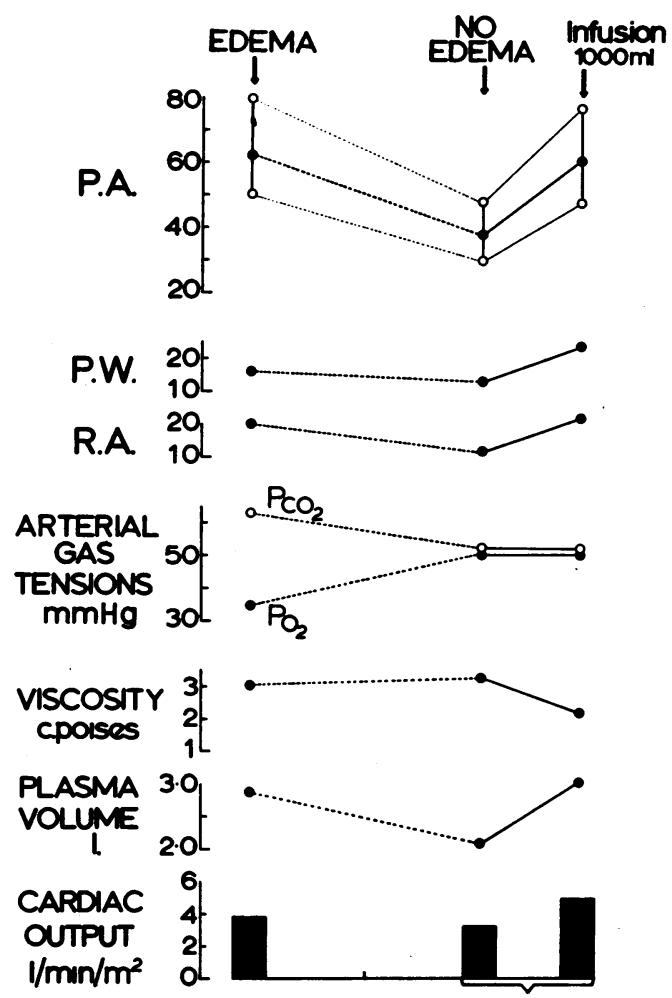

Fig. 6. EFFect in 1 Patient of spontaneous fall in PLASMA VOLUME ON THE PRESSURES (MILliMETERS HG) IN THE PULMONARY ARTERY, PULMONARY WEDGE, AND RIGHT ATRIUM, AND ON ARTERIAL BLOOD GAS TENSIONS, BLOOD VISCOSITY, PLASMA VOLUME, AND CARDIAC OUTPUT.

failure had disappeared and the weight had remained constant for 4 days.

Recovery was associated with a reduction in plasma volume and a fall in pulmonary arterial pressure and in pulmonary vascular resistance, but not to normal levels. On the occasion of the second study, an infusion of $1,000 \mathrm{ml}$ of a solution of HSA was given, this being approximately equal to the amount by which the plasma volume had diminished between the first and second study. This infusion led to a rise in pulmonary arterial, pulmonary wedge, and right atrial pressures and an increase in cardiac output, but pulmonary vascular resistance did not change significantly.

\section{Discussion}

It must be emphasized in considering these results that the patients in the principal study were highly selected and severely disabled. In our experience of large numbers of patients with chronic 
bronchitis and emphysema, those with such severe polycythemia are quite uncommon, so that the findings cannot be regarded as typical for the general population of patients with this disease. On the other hand, the group studied was homogeneous in most respects, and the data are therefore of value when considering the effects of disturbances of specific physiological parameters and of the interactions between them in such patients.

It is normally assumed that polycythemia in patients with chronic bronchitis is due to hypoxemia $(1,8)$, and a significant correlation has been shown between red cell volume and arterial oxygen saturation, both previously $(8,9)$ and in the present study. Administration of oxygen for 6 weeks has been shown capable of restoring hematocrit and red cell volume to normal levels in initially polycythemic patients (35). The presence of a plasma erythropoietic factor has been demonstrated in patients with chronic bronchitis with persistent hypoxemia $(36,37)$ and also in dwellers at high altitude (38). Although a correlation between arterial carbon dioxide tension and red cell volume has been demonstrated by Blumenthal and Cander (11) and in the present study, it appears unlikely that hypercapnia stimulates erythropoiesis, since the level of $\mathrm{Pa}_{\mathrm{CO}_{2}}$ is low in high altitude dwellers (39) and in patients with cyanotic heart disease (40). High levels of carbon dioxide may even inhibit bone marrow activity (41).

The blood viscosity in the main group of patients studied was greatly increased, but fell to normal levels after venesection. We recognize that there are limitations to in vitro methods of measuring the viscosity of blood and that values so determined may not be applicable to the situation where blood is flowing rapidly through small vessels. We believe that the results do, nevertheless, provide an index of the change in viscosity of blood that helps in the understanding of pressure-flow relationships in the pulmonary circulation.

In resting patients after venesection there was a significant fall in pulmonary arterial mean pressure and in pulmonary vascular resistance, but no significant fall in cardiac output. When the factor of blood viscosity was removed from the calculated pulmonary vascular resistance, the quantity termed "pulmonary vascular hindrance" was obtained, which describes the resistance to blood flow due to the dimensions of the pulmonary vascular bed (23). This quantity was significantly larger after venesection than before, suggesting that in the polycythemic state, the pulmonary vascular bed may have been distended, so lowering the resistance to blood flow. We have no experimental evidence to explain this increase in hindrance after venesection. It is possible that nervous reflex mechanisms led to vasodilatation when raised blood volume activated stretch receptors. Alternatively, the effect of the expanded blood volume may have been to dilate, passively, the pulmonary blood vessels. It should be noted that similar changes in hindrance also occurred in the systemic circulation.

It is clear that pulmonary arterial pressure fell more in some patients than in others after venesection. The extent of the fall was related to the size of the reduction in total blood volume, and in those patients in whom it was measured, to the extent of the reduction in central blood volume as well. Substantial falls in pulmonary arterial pressure occurred in those patients with large reductions in total blood volume, but in the patients with little or no change in blood volume, the pressure remained unaltered. Since blood viscosity was approximately halved in all of these patients, it appears that this factor played an unimportant part in determining the level of pulmonary arterial pressure.

We concluded that the resistance to blood flow in the pulmonary vascular bed was increased in these patients, as indicated by the increased pulmonary vascular hindrance. When polycythemia developed, the pulmonary arterial pressure, already raised, increased further due to the increased total blood volume and the increased central blood volume. Any trivial effect that the increased blood viscosity may have had in raising the pressure still further was mitigated by the greater influence of the reduced resistance to blood flow due to the dimensions of the vascular bed. For these reasons, venesection did not have a large effect upon pulmonary arterial pressure, and then only where it led to a fall in blood volume.

The increased blood volume was presumably associated with a rise in central blood volume, and this led to an elevation of the static pressure in the pulmonary circulation. This is supported by 
the observation that although pulmonary wedge pressure did not change significantly in the group as a whole, there were appreciable falls in the patients whose blood volume fell after venesection. Right atrial pressure decreased more consistently irrespective of the extent of change in blood volume.

In an attempt to study further the influence of variations in blood volume, we observed the effects of rapid expansion and reduction produced by infusion and rapid venesection, respectively. Although these procedures had the predicted effects upon pulmonary arterial pressure, there were also changes in cardiac output and large changes in pulmonary wedge pressure that were not seen when blood volume was altered more slowly by repeated venesections. In 1 patient, it was possible to compare the effects of a spontaneous reduction in blood volume over several days with a subsequent acute alteration in blood volume of a similar size. Comparable changes in pulmonary arterial and pulmonary venous pressures after infusions have been observed in normal subjects and in patients with heart disease, and they have been attributed to an increased volume of blood between the pulmonary artery and left atrium (42, 43). De Freitas and his colleagues (44) were unable to show such an increase in pulmonary blood volume and postulated that the acute expansion of blood volume causes pulmonary vasoconstriction. In the present experiments we did not measure pulmonary blood volume. Furthermore, there was an increase in cardiac output after rapid infusion, which makes it even more difficult to state with certainty the mechanism of the rise in pulmonary arterial pressure.

The different behavior of pulmonary wedge pressure, when the blood volume changed gradually instead of rapidly, is not fully explained but may reflect the distribution of pulmonary blood volume, as between the pulmonary arterial and pulmonary venous segments, in the two different circumstances. The different effects of acute and chronic changes in blood volume upon the cardiac output might be similarly explained, a preferential increase in pulmonary venous volume raising the ventricular filling pressure and hence the cardiac output.

The cardiac output at rest in these patients fell within the normal range and was not altered after repeated venesection. This is consistent with the findings of Wade and Bishop (45), who reviewed published data for a large group of patients with chronic bronchitis and emphysema. There is no evidence of a high output state in these patients even though most had recently recovered from an episode of congestive failure, and in some the right atrial pressure was still raised.

The main group of patients in the present study had unusually severe pulmonary hypertension when compared to values found in a large group of patients with chronic bronchitis and emphysema $(10,12,45,46)$. The cause of the pulmonary hypertension in this disease remains uncertain, but the present observations suggest that expansion of the total blood volume is one important factor, whereas increased viscosity is relatively unimportant. Structural changes in pulmonary arterioles, with increase in circular smooth muscle fibers and narrowing of the lumen, have been demonstrated in some patients, and these changes have been correlated with the presence of right ventricular hypertrophy (47). In a small proportion of patients, the pulmonary wedge pressure is raised (48), and this may make a small contribution to the pulmonary hypertension.

Several workers have demonstrated a negative correlation between the percentage oxyhemoglobin saturation of arterial blood and the pulmonary arterial pressure and have speculated as to whether the two are causally related $(1,45,46,49)$. The relatively small effect of oxygen breathing on pulmonary arterial pressure argues against vasoconstriction due to low oxygen tension being a major factor in the pulmonary hypertension of patients studied when free of congestive failure. Similar considerations apply to the positive correlations between arterial carbon dioxide tension and pulmonary arterial pressure. Although it is known that alterations in the blood gases do influence pulmonary arterial pressure in acute experiments, it seems most likely that the observed correlations signify that in certain forms of emphysema, there is severe interference with gas exchange and damage to the pulmonary vascular bed. Both effects are manifestations of one particular form of the disease, and both may be exaggerated during episodes of congestive failure.

The response of cardiac output to exercise was normal in several of these patients, but it was im- 
paired in a larger proportion than in other reported groups of patients with chronic bronchitis and emphysema $(10,12,45)$. Large increases in pulmonary arterial pressure occurred, probably due to the restricted size of the pulmonary vascular bed. Other factors that may have contributed are the increased arterial hypoxemia and hypercapnia and an increase in central blood volume, which has been shown to accompany exercise in the supine position (50). Pulmonary wedge pressure increased abnormally in some patients, possibly due to an increase in intrapleural pressure associated with increased ventilation. Wedge pressure increased also after repeated venesection and so cannot be attributed to an increased blood volume. After venesection, pulmonary arterial pressure did not reach such high levels during exercise, and the response of cardiac output was often improved. The latter might be due to improved function of the right ventricle consequent upon a reduction of the resistance to blood flow, which presumably resulted from the reduction in blood viscosity, or it may indicate that, with a fall in filling pressure, the right ventricle may have been operating on a different part of its function curve.

All of the patients showed a fall in arterial oxygen tension and a rise in carbon dioxide tension with exercise. Total pulmonary ventilation increased to an approximately normal extent, with an increase in respiratory rate. Since the distribution of both ventilation and perfusion must have been grossly disturbed in these patients, it is evident that total alveolar ventilation cannot have increased to a normal extent during exercise and that the average alveolar oxygen tension fell and carbon dioxide tension rose. This may well ac. count for most of the blood gas changes during exercise, although it is possible that an increased abnormality of distribution or a decreased transit time of blood through a reduced capillary bed $(51,52)$ may have played a part.

The serial observations in 2 of the patients are of interest in demonstrating the persistence of the polycythemia, the progressive rise in pulmonary arterial pressure and pulmonary vascular resistance, and the associated fall in cardiac output. Arterial oxygen saturation also fell and, for these individual patients, showed the same negative correlation with pulmonary arterial pressure that was observed in the group as a whole.
The severity of the disease process in these patients is further indicated by the fact that 11 of the 14 patients died during the subsequent 5 years. Repeated venesection did not lead to any improvement in the majority, but a few gained symptomatic benefit. These patients were relieved of headache and dizziness and felt less fatigued, but it is uncertain whether this relief had any organic basis. We did not observe any harmful effect from this line of treatment, but since any beneficial effects are small, it cannot play an important part in the management of such patients.

\section{Summary}

In a highly selected and severely disabled group of patients with chronic bronchitis complicated by marked polycythemia and increased blood viscosity, persistent pulmonary hypertension and severe disturbance in arterial gas tensions were present at rest, whereas cardiac output was normal. During exercise the cardiac output response was impaired in many patients, and pulmonary arterial pressure increased considerably.

After repeated venesection had restored the hematocrit and blood viscosity to normal, there was on the average only a small fall in pulmonary arterial pressure at rest. Individual changes in pulmonary arterial mean pressure correlated positively with changes in total blood volume. The response of cardiac output to exercise improved in several of the patients.

Significant correlations between pulmonary arterial mean pressure, arterial blood gas tensions, and total blood volume were observed at rest and during exercise, both before and after repeated venesection.

Serial observations extending over several years indicate that in such patients the severity of the pulmonary hypertension and the disturbances in the blood gases increase progressively and the prognosis is poor.

In another group of patients without polycythemia, the blood volume was acutely increased by an infusion of human serum albumen. There was a rise in pulmonary arterial and wedge pressures and an increase in cardiac output but no change in pulmonary vascular resistance.

We conclude that the level of total and central blood volume contributes importantly to the pulmonary hypertension in patients with chronic 
bronchitis, whereas increased blood viscosity has little effect.

\section{Acknowledgments}

We wish to thank Staff Nurse Margaret Booth, Mrs. Pauline Furness, Mrs. Anne Hollyhock, and Miss Susan Pepper for their valued technical assistance, and Mrs. K. Farrington for help with parts of the statistical analysis. We are grateful to our many colleagues who have referred patients to us for investigation. We acknowledge with gratitude the financial support received from the Wellcome Trust, the British Heart Foundation, and the Endowment Research Fund of the United Birmingham Hospitals.

\section{References}

1. Cournand, A. Some aspects of the pulmonary circulation in normal man and in chronic cardiopulmonary diseases. Circulation 1950, 2, 641 .

2. Fishman, A. P., and D. W. Richards. The management of cor pulmonale in chronic pulmonary disease, with particular reference to the associated disturbances in the pulmonary circulation. Amer. Heart J. 1956, 52, 149.

3. Motley, H. L. The mechanisms of chronic pulmonary heart disease (cor pulmonale), with and without arterial hypoxemia. Progr. cardiovasc. Dis. 1958$59,1,326$.

4. Ferrer, M. I., and R. M. Harvey. Decompensated pulmonary heart disease with a note on the effect of digitalis in Pulmonary Circulation, W. Adams and I. Veith, Eds. New York, Grune \& Stratton, 1958, p. 171.

5. Cherniack, R. M., and L. Cherniack. Respiration in Health and Disease. Philadelphia, W. B. Saunders, 1961, pp. 173, 293.

6. Roos, A. Poiseuille's law and its limitations in vascular systems in Progress in Research in Emphysema and Chronic Bronchitis, R. F. Grover, Ed. Basel, Karger, 1963, vol. 1, p. 32.

7. Baldwin, E. deF., A. Cournand, and D. W. Richards. Pulmonary insufficiency. III. A study of 122 cases of chronic pulmonary emphysema. Medicine (Baltimore) 1949, 28, 201.

8. Shaw, D. B., and T. Simpson. Polycythaemia in emphysema. Quart. J. Med. 1961, 30, 135.

9. Vanier, T., M. J. Dulfano, C. Wu, and J. F. Desforges. Emphysema, hypoxia and the polycythemic response. New Engl. J. Med. 1963, 269, 169.

10. Kitchin, A. H., C. P. Lowther, and M. B. Matthews. The effects of exercise and of breathing oxygenenriched air on the pulmonary circulation in emphysema. Clin. Sci. 1961, 21, 93.

11. Blumenthal, W. S., and L. Cander. Emphysema in Aging of the Lung, Perspectives, L. Cander and J. H. Moyer, Eds. New York, Grune \& Stratton, 1964, p. 302.

12. Shaw, D. B., R. F. Grover, J. T. Reeves, and G. Blount, Jr. Pulmonary circulation in chronic bronchitis and emphysema. Brit. Heart J. 1965, 27,674 .

13. Goodwin, J. F., and Z. H. Abdin. The cardiogram of congenital and acquired right ventricular hypertrophy. Brit. Heart J. 1959, 21, 523.

14. Sterling, K., and S. J. Gray. Determination of the circulating red cell volume in man by radioactive chromium. J. clin. Invest. 1950, 29, 1614.

15. Mollison, P. L., and N. Veall. The use of the isotope ${ }^{51} \mathrm{Cr}$ as a label for red cells. Brit. J. Haemat. 1955, 1,62 .

16. Pirofsky, B. The determination of blood viscosity in man by a method based on Poiseuille's law. J. clin. Invest. 1953, 32, 292.

17. Wade, O. L., J. M. Bishop, G. Cumming, and K. W. Donald. A method for the rapid estimation of the percentage oxygen saturation and oxygen content of blood. Brit. med. J. 1953, 2, 902.

18. Kopelman, H., and G. de J. Lee. The intrathoracic blood volume in mitral stenosis and left ventricular failure. Clin. Sci. 1951, 10, 383.

19. Hamilton, W. F., J. W. Moore, J. M. Kinsman, and R. G. Spurling. Studies on the circulation. IV. Further analysis of the injection method, and of changes in hemodynamics under physiological and pathological conditions. Amer. J. Physiol. 1932, 99, 534.

20. Bishop, J. M., A. C. Pincock, A. Hollyhock, J. Raine, and R. B. Cole. Factors affecting the measurement of the partial pressure of oxygen in blood using a covered electrode system. Resp. Physiol. 1966, 1, 225.

21. Astrup, P. A simple electrometric technique for the determination of carbon dioxide tension in blood and plasma, total content of carbon dioxide in plasma, and bicarbonate content in "separated" plasma at a fixed carbon dioxide tension $(40 \mathrm{~mm}$ $\mathrm{Hg}$ ). Scand. J. clin. Lab. Invest. 1956, 8, 33.

22. Dexter, L., J. L. Whittenberger, F. W. Haynes, W. T. Goodale, R. Gorlin, and C. G. Sawyer. Effect of exercise on circulatory dynamics of normal individuals. J. appl. Physiol. 1951, 3, 439.

23. Lamport, $H$. Haemodynamics in Textbook of Physiology, 17th ed., J. F. Fulton, Ed. Philadelphia, W. B. Saunders, 1955, p. 589.

24. Yiengst, M. J., and N. W. Shock. Blood and plasma volume in adult males. J. appl. Physiol. 1962, 17, 195.

25. Hart, D., and J. Metz. The estimation of red cell volume with ${ }^{\circ} \mathrm{Cr}$-labelled erythrocytes and plasma volume with radioiodinated human serum albumin. J. clin. Path. 1962, 15, 459.

26. Wennesland, R., E. Brown, J. Hopper, Jr., J. L. Hodges, Jr., O. E. Guttentag, K. G. Scott, I. N. Tucker, and B. Bradley. Red cell, plasma and blood volume in healthy men measured by radiochromium $\left(\mathrm{Cr}^{\mathrm{a}}\right)$ cell tagging and hematocrit: influence of age, somatotype and habits of physical activity on the variance after regression of vol- 
umes to height and weight combined. J. clin. Invest. 1959, 38, 1065.

27. Brandfonbrener, M., M. Landowne, and N. W. Shock. Changes in cardiac output with age. Circulation 1955, 12, 557.

28. Granath, A., B. Jonsson, and T. Strandell. Circulation in healthy old men, studied by right heart catheterization at rest and during exercise in supine and sitting position. Acta med. scand. 1964, $176,425$.

29. Dexter, L., F. W. Haynes, and G. T. Smith. Physiologic changes in the pulmonary circulation with age in Aging of the Lung, Perspectives, L. Cander and J. H. Moyer, Eds. New York, Grune \& Stratton, 1964, p. 194.

30. Segel, N., W. A. Hudson, P. Harris, and J. M. Bishop. The circulatory effects of electrically induced changes in ventricular rate at rest and during exercise in complete heart block. J. clin. Invest. 1964, 43, 1541.

31. Doyle, J. T., J. S. Wilson, C. Lépine, and J. V. Warren. An evaluation of the measurement of the cardiac output and of the so-called pulmonary blood volume by the dye-dilution method. J. Lab. clin. Med. 1953, 41, 29.

32. Lilienfield, L. S., R. D. Kovach, P. A. Marks, L. M. Hershenson, G. P. Rodnan, F. G. Ebaugh, Jr., and E. D. Freis. The hematocrit of the lesser circulation in man. J. clin. Invest. 1956, 35, 1385.

33. Donald, K. W., J. M. Bishop, G. Cumming, and O. L. Wade. The effect of exercise on the cardiac output and circulatory dynamics of normal subjects. Clin. Sci. 1955, 14, 37.

34. Raine, J. M., and J. M. Bishop. A-a difference in $\mathrm{O}_{2}$ tension and physiological dead space in normal man. J. appl. Physiol. 1963, 18, 284.

35. Chamberlain, D. A., and F. J. C. Millard. The treatment of polycythaemia secondary to hypoxic lung disease by continuous oxygen administration. Quart. J. Med. 1963, 32, 341.

36. Linman, J. W., and F. H. Bethell. Plasma erythropoietic-stimulating factor in man: observations on patients with polycythaemia vera and secondary polycythaemia. J. Lab. clin. Med. 1957, 49, 113.

37. Noyes, W. D., B. M. Domm, and L. C. Willis. Erythropoietin assay in refractory anemia and polycythemia. Clin. Res. 1962, 10, 27.

38. Merino, C. F. Plasma erythropoietic factor in polycythaemia of high altitudes. U. S. Air Force School of Aviation Medicine Report 56-103, 1956, p. 1.

39. Hurtado, A., and H. Aste-Salazar. Arterial blood gases and acid-base balance at sea level and at high altitudes. J. appl. Physiol. 1948, 1, 304.
40. Bing, R. J., L. D. Vandam, J. C. Handelsman, J. A. Campbell, R. Spencer, and H. E. Griswold. Physiological studies in congenital heart disease. VI. Adaptations to anoxia in congenital heart disease ,with cyanosis. Bull. Johns Hopk. Hosp. 1948, 83, 439.

41. Ratto, O., W. A. Briscoe, J. W. Morton, and J. H. Comroe. Anoxemia secondary to polycythemia and polycythemia secondary to anoxemia. Amer. J. Med. 1955, 19, 958.

42. Witham, A. C., J. W. Fleming, and W. L. Bloom. The effect of the intravenous administration of dextran on cardiac output and other circulatory dynamics. J. clin. Invest. 1951, 30, 897.

43. Oakley, C., G. Glick, M. N. Luria, B. F. Schreiner, Jr., and P. N. Yu. Some regulatory mechanisms of the human pulmonary vascular bed. Circulation 1962, 26, 917.

44. De Freitas, F. M., E. Z. Faraco, D. F. De Azevedo, J. Zaduchliver, and I. Lewin. Behavior of normal pulmonary circulation during changes of total blood volume in man. J. clin. Invest. 1965, 44, 366.

45. Wade, O. L., and J. M. Bishop. Cardiac Output and Regional Blood Flow. Oxford, Blackwell, 1962, p. 149.

46. Enson, Y., C. Giuntini, M. L. Lewis, T. Q. Morris, M. I. Ferrer, and R. M. Harvey. The influence of hydrogen ion concentration and hypoxia on the pulmonary circulation. J. clin. Invest. 1964, 43, 1146.

47. Hicken, P., D. Heath, and D. B. Brewer. The relation between the weight of the right ventricle and the percentage of abnormal airspace in the lung in emphysema. J. Path. Bact. 1966, in press.

48. Herles, F., S. Daum, and B. Bednor. The cardiopulmonary failure with elevated wedge pressure in pulmonary emphysema. Proceedings of the Third European Congress on Cardiology. 1960, 2A., p. 491.

49. Harris, P., and D. Heath. The Human Pulmonary Circulation. Edinburgh, E. and S. Livingstone, 1962, p. 279.

50. Braunwald, E., and E. R. Kelly. The effects of exercise on central blood volume in man. J. clin. Invest. 1960, 39, 413.

51. Williams, M. H., Jr., and L. R. Zohman. Cardiopulmonary function in chronic obstructive emphysema. Amer. Rev. resp. Dis. 1959, 80, 689.

52. Rossier, P. H., A. A. Bühlmann, and K. Wiesinger. Transfer of respiratory gas from the alveolus into the blood in Respiration: Physiologic Principles and Their Clinical Applications, P. C. Luchsinger and K. M. Moser, Eds. St. Louis, C. V. Mosby, 1960, p. 82. 